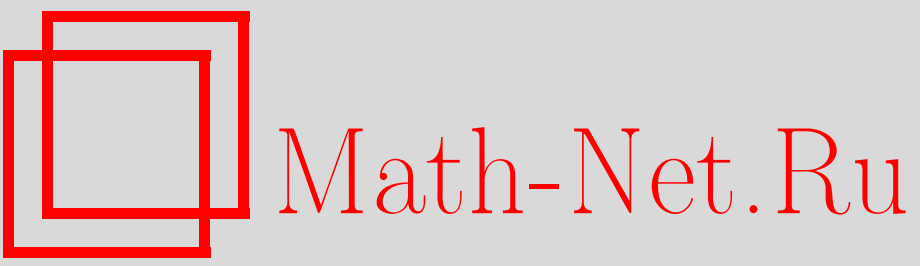

П. Свенссон, Динамические системы в неразветвленных и тотально разветвленных расширениях p-адического поля, Изв. РАН. Сер. матем., 2005, том 69, выпуск 6, 211-218

DOI: https://doi.org/10.4213/im673

Использование Общероссийского математического портала Math-Net.Ru подразумевает, что вы прочитали и согласны с пользовательским соглашением

http://www.mathnet.ru/rus/agreement

Параметры загрузки:

IP: 54.224 .187 .69

26 апреля 2023 г., 18:27:02 


\section{Динамические системы в неразветвленных и тотально разветвленных расширениях p-адического поля}

\footnotetext{
Использована теория локальных полей для изучения специального класса дискретных динамических систем, для которых итерируемая функция - полином, коэффициенты которого принадлежат кольцу целых $\mathfrak{p}$-адического поля.

Библиография: 17 наименований.
}

\section{§1. Введение}

В последние десять лет к теории динамических систем над полем $p$-адических чисел проявлялся постоянно растущий интерес (см., например, [1], [2], [4], [12]-[14]), обусловленный исследованиями, проводимыми в областях $p$-адической математической физики [16], [17], [6] и когнитивных наук [8]. Для получения результатов о $p$-адических динамических системах часто используется теория чисел (см., например, [9]). В настоящей работе мы будем использовать теорию локальных полей (см., например, [3], [5], [10], [15]) для изучения специального класса динамических систем, для которых итерируемая функция - полином с коэффициентами, лежащими в кольце целых поля p-адических чисел.

\section{§2. Обозначения}

Всюду далее $k-\mathfrak{p}$-адическое поле, т. е. локальное поле характеристики нуль. Локальное поле по определению полно относительно нетривиального дискретного нормирования и имеет конечное поле вычетов.

Обозначим через | | норму на $k$ и через $\mathfrak{V}_{k}$ группу значений $k$, т. е. множество норм всех ненулевых элементов в $k$. Для заданных $a \in k$ и $r>0$ будем использовать следующие обозначения:

$$
\begin{aligned}
& B_{r}(a, k)=\{x \in k:|x-a|<r\}, \\
& \bar{B}_{r}(a, k)=\{x \in k:|x-a| \leqslant r\}, \\
& \bar{S}_{r}(a, k)=\{x \in k:|x-a|=r\}
\end{aligned}
$$

для открытого, замкнутого шара и сферы соответственно с центром в $a$ и радиусом $r$. Заметим, что так как нормирование дискретное и, таким образом, неархимедово, то $B_{r}(a, k)$ и $\bar{B}_{r}(a, k)$ - одновременно открытые и замкнутые множества в топологии, индуцированной || , и $S_{r}(a, k)$ пусто, если $r \notin \mathfrak{V}_{k}$. Следовательно, если $r \notin \mathfrak{V}_{k}$ и $s=\max \left\{\rho \in \mathfrak{V}_{k}: \rho<r\right\}$, то $B_{r}(a, k)=\bar{B}_{s}(a, k)$.

Кольцо целых в поле $k$ мы будем обозначать через o и соответствуюший максимальный идеал - через p. Обозначим через $k_{\mathfrak{p}}$ поле вычетов поля $k$, т. е. $k_{\mathfrak{p}}=\mathfrak{o} / p$. Поскольку нормирование дискретное, $\mathfrak{p}$ - главный идеал. Образующая $\pi$ идеала $\mathfrak{p}$ называется простым элементом в поле $k$. Для каждого простого $\pi$ элемент $|\pi|$ 
порождает группу значений $\mathfrak{V}_{k}$, и, обратно, если $|\pi|$ порождает $\mathfrak{V}_{k}$ и $|\pi|<1$, то $\pi$ порождает p. Рассмотрим простой элемент $\pi$. Каждый ненулевой элемент $a \in k$ может быть записан как $a=u \pi^{n}$ для некоторой единищы $u \in \mathfrak{o}$, причем $n$ не зависит от $\pi$. Назовем $n$ порядком $a \in k$ относительно $\pi$ и запишем ${ }^{1} n=\operatorname{ord}_{\pi}(a)$. Все конечные расширения $k$ предполагаются вложенными в фиксированное алгебраическое замыкание $K$ поля $k$. Если $L$ - конечное расширение $k$, то нормирование на $k$ может быть продолжено (одним и только одним способом) до нормирования на $L$ (которое мы также будем обозначать через | |) так, что $L$ также будет являться $\mathfrak{p}$-адическим полем. Используем обозначения $\mathfrak{V}_{L}, \mathfrak{O}, \mathfrak{P}$ и $L_{\mathfrak{P}}$ для группы значений, кольца целых, максимального идеала и поля вычетов соответственно.

Если $F$ - некоторое поле, мы будем использовать обозначение $F^{*}$ для его мультипликативной групшы. Конечное поле, состояшее из $r$ элементов, где $r$ - степень простого числа, будем обозначать через $\mathbb{F}_{r}$.

\section{§3. Ветвление}

Напомним некоторые понятия и теоремы, связанные с ветвлением конечных расширений $\mathfrak{p}$-адических полей. Если $L$ - конечное расширение $k$, то $\mathfrak{V}_{k}-$ подгруппа в $\mathfrak{V}_{L}$. Мы используем запись

$$
e=e(L / k)=\left(\mathfrak{V}_{L}: \mathfrak{V}_{k}\right)
$$

для так называемого индекса ветвления расширения $L / k$. Более того, поле вычетов $k_{\mathfrak{p}}$ поля $k$ может быть вложено в поле вычетов $L_{\mathfrak{P}}$ поля $L$. Под относительной степенью расширения $L / k$ мы понимаем число

$$
f=f(L / K)=\left[L_{\mathfrak{P}}: k_{\mathfrak{p}}\right] .
$$

Числа $e$ и $f$ связаны друг с другом согласно следующей хорошо известной теореме.

ТЕОРема 3.1. Пусть $k$ - p-адическое поле $и L$ - конечное расширение $k$. Тогда

$$
[L: k]=e(L / k) f(L: k) .
$$

Конечное расширение называется неразветвленным, если $e=1$ (и, таким обpaзом, $f=[L: k])$. С другой стороны, если $e=[L: k]$ (следовательно, $f=1)$, то $L / k$ называется тотально разветвленнылм.

Сформулируем две хорошо известные теоремы, которые описывают, как строить неразветвленные и тотально разветвленные расширения $\mathfrak{p}$-адического поля $k$.

ТЕОрема 3.2. Пусть $m(x) \in k_{\mathfrak{p}}[x]$ - неприводимый полином степени $n$. Предположим, что $\widehat{m}(x) \in \mathfrak{o}[x]$ той же степени, что и $m(x)$, и канонически отображается в $m(x)$. Тогда $\widehat{m}(x)$ неприводим над $k u L=k[x] /\langle\widehat{m}(x)\rangle$ -

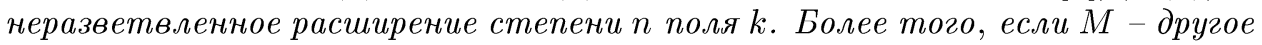
неразветвленное расширение степени $n$, то $M$ изоморфно $L$.

Теорема 3.3. Предположим, что $L / k$ - тотально разветвленное расиирение степени $n$. Тогда $L=k(\alpha)$ для некоторого корня многочлена Эйзенштейна, т.е. многочлена

$$
f(x)=a_{0}+a_{1} x+\cdots+a_{n-1} x^{n-1}+x^{n} \in k[x]
$$

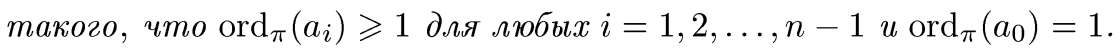

\footnotetext{
${ }^{1}$ Будем использовать соглашение: $\operatorname{ord} \pi(0)=\infty$.
} 
Обратно, если $L=k(\beta)$ - расширение $k$, получаемое присоединение корня $\beta$ полинома Эйзенштейна степени $n$ из $k[x]$, то $L / k$ - тотально разветвленное накрытие степени $n$.

Доказательство трех теорем этого параграффа можно найти, например, в [3].

\section{§4. О динамических системах}

Пусть $L$ - конечное расширение $\mathfrak{p}$-адического поля $k$. Для заданного многочлена $h(x)$ в $k[x]$ мы определяем соответствующую дискретную динамическую систему (или, для краткости, динамическую систему) $h$ на $L$ как отображение $L \ni \beta \rightarrow$ $h(\beta) \in L$.

Если $h$ - динамическая система и $\alpha$ - элемент $L$, мы определяем последовательность $\left(\alpha_{j}\right)_{j=0}^{\infty}$ в $L$ рекурсивно:

$$
\begin{aligned}
\alpha_{0} & =\alpha, \\
\alpha_{j} & =h^{j}\left(\alpha_{j-1}\right), \quad j=1,2, \ldots .
\end{aligned}
$$

Будем записывать $h^{j}(\alpha)=\alpha_{j}$. Здесь $h^{j}$ может быть интерпретировано как $j$-кратная итерация отображения $h$.

ОПРЕДЕЛЕНИЕ 4.1. Пусть $h$ - динамическая система на $L$. Тогда $\alpha \in L$ называется неподвижной точкой $h$, если $h(\alpha)=\alpha$. Неподвижная точка $\alpha$ динамической системы $h$ называется:

(i) притягивающей точкой $h$, если $\left|h^{\prime}(\alpha)\right|<1$;

(ii) безразличной точкой $h$, если $\left|h^{\prime}(\alpha)\right|=1$.

Здесь $h^{\prime}(x)$ обозначает производную $h(x)$.

Поведение точек в окрестности неподвижной точки описывается следующей теоремой (см. [7]).

ТЕОремА 4.2. Пусть $\alpha$ - неподвижная точка динамической системы $h$ на $L$.

(i) Если $\alpha$ - притягивающая точка, то существует окрестность $V$ точки $\alpha$ такая, что $\lim _{j \rightarrow \infty} h^{j}(\beta)=\alpha$ для любой $\beta \in V$.

(ii) Если $\alpha$ - нейтральная точка, то найдется иар $B_{r}(\alpha, L)$ такой, что каждая сфера $S_{\rho}(\alpha, L)$, де $\rho<r$, инвариантна относительно динамической системы $h$, m. е. $h\left[S_{\rho}(\alpha, L)\right] \subseteq S_{\rho}(\alpha, L)$.

Используя утверждения (i) и (ii) теоремы 4.2, введем следующие определения.

ОПРЕДЕЛЕНИЕ 4.3. Пусть $\alpha$ - неподвижная точка динамической системы $h$ на $L$.

(i) Если $\alpha-$ притягиваюшая точка, то

$$
A(\alpha, L)=\left\{\beta \in L: \lim _{j \rightarrow \infty} h^{j}(\beta)=\alpha\right\}
$$

называется притягивающим бассейном точки $\alpha$ в $L$ относительно $h$.

(ii) Если $\alpha$ - нейтральная точка динамической системы $h$, то шар $B_{r}(\alpha, L)$ такой, что каждая сфера $S_{\rho}(\alpha, L)$, для которой $\rho<r$, инвариантна относительно $h$ и называется диском Зигеля точки $\alpha$ в $L$ относительно $h$. Объединение всех дисков Зигеля назьвается максимальным диском Зигеля точки $\alpha$ в $L$ относительно $h$ и обозначается $\operatorname{Si}(\alpha, L)$. 
ЗАмЕЧАНИЕ 4.4. По определению максимальный диск Зигеля является шаром $B_{s}(\alpha, L)$ для некоторого $s>0$, поэтому любое семейство дисков Зигеля $\left\{B_{r_{i}}(\alpha, L)\right\}_{i \in J}$ упорядочено по включению. Однако если $s \notin \mathfrak{V}_{L}$ и $r=\max \{\rho \in$ $\left.\mathfrak{V}_{L}: \rho<s\right\}$, то $\operatorname{Si}(\alpha, L)=\bar{B}_{r}(\alpha, L)$.

Следующая теорема доказана в [7].

ТеОрема 4.5. Пусть $h$ - динамическая система на $L$ и $\alpha$ - неподвижная точка системы $h$. Пусть $r$ - положительное действительное число.

(i) Если $\alpha$ - притягивающая точка и $r$ удовлетворяет неравенству

$$
\max _{n \geqslant 2}\left(\left|\frac{h^{(n)}(\alpha)}{n !}\right| r^{n-1}\right)<1
$$

mо $\bar{B}_{r}(\alpha, L) \subseteq A(\alpha, L)$. Здесь $h^{(n)}-n$-я производная $h$.

(ii) Если $\alpha$ - безразличная точка и $r$ удовлетворяет $(4.1)$, то $\bar{B}_{r}(\alpha, L) \subseteq$ $\operatorname{Si}(\alpha, L)$.

Поскольку $h$ - динамическая система и $h(x)$ - нормированный полином с коэффициентами в кольце о целых поля $k$, нам понадобится следующая лемма.

ЛЕмма 4.6. Пусть $h[x] \in \mathfrak{o}[x]$ - полином степени $m u \alpha$ - некоторый элемент в (расширении) $k$ такой, что $|\alpha| \leqslant 1$. Предположим, что стариий коэффициент $h(x)$ есть единица. $B$ этом случае $r \in \mathbb{R}^{+}$удовлетворяет неравенству (4.1) тогда и только тогда, когда $r<1$.

ДокАЗАТЕЛЬСТво. $(\Rightarrow)$ Если неравенство (4.1) вьполнено, то

$$
\left|\frac{h^{(m)}(\alpha)}{m !}\right| r^{m-1}<1 .
$$

Однако $h^{(m)}(\alpha) r^{m-1}=u m$ ! для некоторой единицы $u \in \mathfrak{o}$. Следовательно, $r<1$.

$(\Leftarrow)$ Предположим $r<1$. Полагая $h(x)=b_{m} x^{m}+\cdots+b_{1} x+b_{0}$, где $\left|b_{m}\right|=1$, находим

$$
\frac{h^{(n)}(\alpha)}{n !}=\frac{1}{n !} \sum_{i=0}^{m-n} \frac{(n+i) !}{i !} b_{n+i} \alpha^{i}=\sum_{i=0}^{m-n}\left(\begin{array}{c}
n+i \\
n
\end{array}\right) b_{n+i} \alpha^{i}
$$

для любого $n$ такого, что $2 \leqslant n \leqslant m$. Здесь правая часть целая, значит, имеем

$$
\left|\frac{h^{(n)}(\alpha)}{n !}\right| r^{n-1}<\left|\frac{h^{(n)}(\alpha)}{n !}\right| \leqslant 1,
$$

что доказывает лемму.

\section{§5. Динамические системы в расширениях полей}

Пусть $k-\mathfrak{p}$-адическое поле, и предположим, что его поле вычетов имеет характеристику $p$ и содержит $p^{f}$ элементов.

Всякий раз, когда $a$ принадлежит кольцу цельх в p-адическом поле, будем использовать запись $\bar{a}$ для канонического образа $a$ в поле вычетов. Аналогично будем использовать запись $\bar{g}(x)$ для канонического образа полинома, коэффициенты которого целые. 
Предположим, что $g(x) \in[o](x)$ - приведенный неприводимый многочлен степени $q, q$ - простое, и положим $L=k(\alpha)$ для некоторого корня $\alpha$ в $g(x)$. Тогда расширение $L / k$ будет либо неразветвленным, либо тотально разветвленным (по теореме 3.1). Далее, множество всех неподвижных точек динамической системы

$$
h(x)=x+g(x)
$$

совпадает с множеством корней многочлена $g(x)$ в $L$. Наша цель - изучить в расширении поля, содержашем неподвижную точку, характер неподвижных точек $h$ и дать описание соответствуюших притягивающих бассейнов или максимальных дисков Зигеля.

5.1. Неразветвленный случай. Справедлива следующая теорема.

TEOPEMA 5.1. Пусть $g(x)=x^{q}+a_{q-1} x^{q-1}+\cdots+a_{1} x+a_{0} \in \mathfrak{o}[x]-$ непрuводимый многочлен простой степени q. Предположим, что $g(x)$ определяет неразветвленное расширение $L / k$ и что $\bar{g}(x) \in k_{\mathfrak{p}}[x]$ неприводим. Тогда каждая неподвижная точка $\alpha \in L$ динамической системы (5.1) является притягивающей, если и только если $q=p, u$

$$
\begin{array}{ll}
a_{1} & \equiv-1 \quad(\bmod \pi), \\
a_{n} & \equiv 0 \quad(\bmod \pi), \quad n=2,3, \ldots, p-1 .
\end{array}
$$

ДокАЗАТЕЛЬСтво. $(\Rightarrow)$ Предположим, что $\alpha-$ притягивающая точка. Тогда $\left|h^{\prime}(\alpha)\right|<1$, откуда следует, что $\bar{h}^{\prime}(\bar{\alpha})=0$ в $L_{\mathfrak{P}}$. Минимальный полином $\bar{\alpha}$ над $k_{\mathfrak{p}}$ есть $\bar{g}(x)$, поэтому $\bar{g}(x) \mid \bar{h}^{\prime}(x)$. Поскольку $\operatorname{deg} \bar{h}^{\prime}(x)<\operatorname{deg} \bar{g}(x)$, получаем противоречие, если только $\bar{h}^{\prime}(x)$ не нулевой полином в $k_{\mathfrak{p}}[x]$. Но в этом случае

$$
q \equiv(q-1) a_{q-1} \equiv(q-2) a_{q-2} \equiv \cdots \equiv 2 a_{2} \equiv a_{1}+1 \equiv 0 \quad(\bmod \pi),
$$

откуда немедленно вытекают равенство $q=p$ и сравнения (5.2).

$(\Leftarrow)$ Если $q=p$ и сравнения (5.2) справедливы, то все коэффициенты $h(x)$, за исключением старшего, делятся на $\pi$ и, так как $\operatorname{deg} h(x)=p$, производная многочлена $\bar{h}(x)$ тождественно равна нулю в $k_{\mathfrak{p}}[x]$. Это означает, в частности, что $\bar{h}^{\prime}(\alpha)=0$ для любой неподвижной точки $\alpha$ динамической системы $h$, или, другими словами, что $\left|h^{\prime}(\alpha)\right|<1$. Следовательно, $\alpha$ - притягивающая точка.

В том случае, когда рассматриваемая неподвижная точка - притягивающая, ее притягивающий бассейн можно определить, используя следующую теорему.

ТЕОрема 5.2. Для заданных $a, b \in \mathfrak{o}$ положим

$$
g_{a, b}(x)=x^{p}+a x+b \in \mathfrak{o}[x] .
$$

Предположим, что $a+1$ не является единичей, $a \sum_{j=1}^{f} b^{p^{j-1}}$ является единицей. Тогда полином $g_{a, b}(x)$ определяет неразветвленное расширение степени р поля $k$. Более того, каждая неподвижная точка соответствующей динамической системь

$$
h_{a, b}(x)=x+g_{a, b}(x)
$$

является притягивающей, и $A(\alpha, L)=B_{1}(\alpha, L)$.

ДокАЗАТЕльство. Сначала докажем, что $g_{a, b}$ неприводим над полем $k$ всякий раз, когда $a+1$ не является единицей, а $\sum_{j=1}^{f} b^{p^{j-1}}-$ единица. Это будет доказано, 
если мы покажем, что полином $\bar{g}_{a, b}=x^{p}-x+\bar{b}$. где $\bar{b} \in k_{\mathfrak{p}}^{*}$ (так как $\bar{b}$ должно быть единицей), неприводим над $k_{\mathfrak{p}}$. Теперь, поскольку $k_{\mathfrak{p}}$ изоморфино $\mathbb{F}_{p}$, мы найдем, что сумма $\sum_{j=1}^{f} b^{p^{j-1}}$ (не равная нулю по предположению) есть абсолютный след $\bar{b}$. Таким образом, полином $\bar{g}_{a, b}(x)$ неприводим над $k_{\mathfrak{p}}$ (см. $[11$, с. 120$\left.]\right)$.

По теореме 3.2 полином $g_{a, b}$ определяет неразветвленное расширение степени $p$, а из теоремы 5.1 следует, что каждая неподвижная точка соответствующей динамической системы является притягивающей. Поскольку $|\alpha|=1$ для каждой неподвижной точки $\alpha$ динамической системы $h_{a, b}$, то из теоремы $4.5,(\mathrm{i})$ и леммы 4.6 следует $B_{1}(\alpha, L) \subseteq A(\alpha, L)$.

Можно легко показать, что неравенство $|\beta-\alpha|>1$ влечет $\left|h_{a, b}(\beta)-\alpha\right|>$ $|\beta-\alpha|$, следовательно, $\beta \notin A(\alpha, L)$. Таким образом, остается показать, что ни один элемент $S_{1}(\alpha, L)$ не принадлежит $A(\alpha, L)$. Предположим противное: можно найти элемент $\beta \in S_{1}(\alpha, L)$ такой, что

$$
\left|h_{a, b}(\beta)-\alpha\right|<1
$$

Канонически отображая $\alpha$ и $h_{a, b}(\beta)$ в поле вычетов $L_{\mathfrak{P}}$, получим условия $\bar{\beta} \neq \bar{\alpha}$ и $\bar{h}_{a, b}(\bar{\beta})=\bar{\alpha}$. Однако отображение $L_{\mathfrak{P}}$ в себя, переводяшее $x$ в элемент $\bar{h}_{a, b}(x)=$ $x^{p}+\bar{b}$, взаимно однозначно. Таким образом, ни один элемент $S_{1}(\alpha, L)$ не принадлежит $A(\alpha, L)$. Теорема доказана.

5.2. Тотально разветвленный случай. Сформулируем лемму, которая будет использоваться при доказательстве основного результата настоящего пункта.

ЛЕмма 5.3. Пусть $m$ и $n$ - положстельные челье числа, и пусть $r=p^{m}$ для некоторого простого числа р. Тогда полином $x^{n}+1$ имеет корень в $\mathbb{F}_{p}$, если и только если $(r-1) /(n, r-1)$ - четное число.

ДокАЗАТЕЛЬСтво. Поскольку $\mathbb{F}_{r}-$ поле разложения $x^{r-1}-1$ над $\mathbb{F}_{p}$, полином $x^{n}+1$ имеет корень в $\mathbb{F}_{r}$ тогда и только тогда, когда результант этих двух полиномов равен нулю. Это доказьвает лемму, так как результант есть $(-1)^{r-1}\left((-1)^{(r-1) / d}-1\right)^{d}$, где $d=(n, r-1)$.

ТЕОРемА 5.4. Пусть $q-$ простое число. Предположим, что

$$
g(x)=x^{q}+a_{q-1} x^{q-1}+\cdots+a_{1} x+a_{0} \in \mathfrak{o}[x]
$$

определяет тотально разветвленное накрытие $L / k$ степени $q$. Пусть $\alpha-$ неподвижная точка динамической системь $h(x)=x+g(x)$. Тогда $\alpha-$ безразличная точка и ее максимальным диском Зигеля $\operatorname{Si}(\alpha, L)$ является шар $B_{|\pi|^{-1 / q}}(\alpha, L)$ тогда и только тогда, когда $p^{f} u\left(p^{f}-1\right) /\left(q-1, p^{f}-1\right)$ нечетные числа, и является шар $B_{1}(\alpha, L)$ в противном случае.

ДокАЗАТЕЛьство. Заметим, что $g(x)$ неприводим. Рассмотрим два случая.

1. Пусть $\pi \mid a_{0}$. Поскольку $g(x)$ неприводим, каждое $a_{i}$ делится на $\pi$. Частным случаем таких полиномов являются полиномы Эйзенштейна (см. теорему 3.3). Более того, если $\alpha$ - нуль полинома $g(x)$ и, следовательно, является неподвижной точкой $h(x)$, то $|\alpha|<1$. Значит, $\alpha$ - безразличная точка, так как

$$
\left|h^{\prime}(\alpha)\right|=\left|1+a_{1}+2 a_{2} \alpha+3 a_{3} \alpha^{2}+\cdots+q \alpha^{q-1}\right|=1 .
$$

Докажем, что максимальным диском Зигеля точки $\alpha$ относительно $h$ является шар $B_{|\pi|^{-1 / q}}(\alpha, L)$. Выберем $\rho \in \mathfrak{V}_{L}$ такое, что $\rho<|\pi|^{-1 / q}$. Так как $L / k-$ 
тотально разветвленное расширение степени $q$, группа значений $\mathfrak{V}_{L}$ есть циклическая группа $\left\langle|\pi|^{-1 / q}\right\rangle=\left\{|\pi|^{i / q}: i \in \mathbb{Z}\right\}$, и мы можем предположить, что $\rho<1$ (см. замечание 4.4).

Из теоремы 4.5 , (ii) и леммы 4.6 следует, что $S_{\rho}(\alpha, L)$ инвариантна относительно $h$, если $\rho<1$.

Случай, когда $\rho=1$, должен быть рассмотрен отдельно, т. е. мы должны выяснить, принадлежит ли $h(\beta)$ сфере $S_{1}(\alpha, L)$, если $\beta \in S_{1}(\alpha, L)$. Мы изучаем каноническую последовательность $\left(\bar{\beta}_{j}\right)_{j=0}^{\infty}$ в поле вычетов $L_{\mathfrak{P}}$. Поскольку мы рассматриваем тотально разветвленное расширение, то $L_{\mathfrak{P}}$ изоморфно $k_{\mathfrak{p}}$. Более того, $\bar{h}(x)=x^{q}+x \in k_{\mathfrak{p}}[x]$. Если $|\beta-\alpha|=1$, то $\bar{\beta} \neq \bar{\alpha}=0$ в $k_{\mathfrak{p}}$. Отсюда вытекает, что $\beta \notin \operatorname{Si}(\alpha, L)$ тогда и только тогда, когда $\bar{h}(\beta)=0$, но $\bar{h}(\beta)=\bar{\beta}^{q}+\bar{\beta}$, и так как $\bar{\beta} \neq 0$, мы получаем, что $h(\beta) \notin S_{1}(\alpha, L)$ тогда и только тогда, когда $\bar{\beta} \in k_{\mathfrak{p}}^{*}-$ корень многочлена $x^{q-1}+1 \in k_{\mathfrak{p}}[x]$. Так как $k_{\mathfrak{p}}$ изоморфно $\mathbb{F}_{p^{f}}$, такой корень сушествует, если $p^{f}$ четно. Если $p^{f}$ нечетно, то такой нуль существует тогда и только тогда, когда $\left(p^{f}-1\right) /\left(q-1, p^{f}-1\right)$ - нечетное число в соответствии с леммой 5.3 .

Очевидно, не существует инвариантных относительно $h$ сфер $S_{\rho}(\alpha, L)$ таких, что $\rho \in \mathfrak{V}_{L}$ и $\rho \geqslant|\pi|^{-1 / q}$. Это завершает доказательство теоремы в рассматриваемом случае.

2 . Пусть $\pi \nmid a_{0}$. В этом случае $\alpha$ есть единица в $\mathfrak{O}-$ кольце целых в $L$. Другими словами, $|\alpha|=1$. Это означает, что существует единственное представление $\alpha$ :

$$
\alpha=\zeta \eta,
$$

где $\zeta \in \mathfrak{o}-\left(p^{f}-1\right)$-й корень из единищы и $\eta \in 1+\mathfrak{P}$. Минимальный многочлен $\eta$ над $\mathfrak{o}$ есть

$$
m(x)=x^{q}+\zeta^{-1} a_{q-1} x^{q-1}+\cdots+\zeta^{-q+1} a_{1} x+\zeta^{-q} a_{0} .
$$

Если мы зафиксируем простое П в $L$ (т. е. образующую идеала $\mathfrak{P})$, то можно при некотором $\gamma \in \mathfrak{O}$ выписать следуюшее представление:

$$
\eta=1+\gamma \Pi \text {. }
$$

Минимальный многочлен $s[x]$ элемента $\gamma$ П имеет степень $q$ и $m(x)=s(x-1)$. Однако

$$
s(x-1)=x^{q}+\frac{s^{(q-1)}(-1)}{(q-1) !} x^{q-1}+\cdots+s^{\prime}(-1) x+s(-1),
$$

откуда с учетом соотношения (5.5) получим

$$
a_{i}=\frac{\zeta^{q-i} s^{(i)}(-1)}{i !}
$$

для любого $i$. Положим

$$
s(x)=x^{q}+b_{q-1} x^{q-1}+b_{q-2} x^{q-2}+\cdots+b_{1} x+b_{0}
$$

для некоторых $b_{i} \in \mathfrak{o}$. Тогда мы найдем, что, так как $|\gamma \Pi| \leqslant|\pi|<1$, каждое $b_{i}$ делится на $\pi$. Следовательно, $\bar{s}(x)=x^{q} \in k_{\mathfrak{p}}[x]$ и, таким образом,

откуда следует

$$
\bar{s}^{(i)}(-1)=\frac{q !}{(q-i) !}(-1)^{q-i}
$$

$$
\bar{h}(x)=\sum_{i=0}^{q}\left(\begin{array}{l}
q \\
i
\end{array}\right)(-\bar{\zeta})^{q-i} x^{i}=(x-\bar{\zeta})^{q} .
$$


Значит,

$$
\bar{h}^{\prime}(\bar{\alpha})=1+q(\bar{\alpha}-\bar{\zeta})^{q-1} .
$$

Однако вследствие (5.4) и (5.6) $\bar{\alpha}=\bar{\zeta}$ в $k_{\mathfrak{p}}$. Следовательно, $\bar{h}^{\prime}(\alpha) \neq 0$, а значит, $\alpha$-нейтральная точка.

Используя теорему 4.5 , (ii) и лемму 4.6 , мы найдем, что каждая сфера $S_{\rho}(\alpha, L)$ при $\rho<1$ инвариантна относительно $h$ и при $\rho \geqslant|\pi|^{-1 / q}$ не инвариантна.

Случай, когда $\rho=1$, также должен быть рассмотрен отдельно. Пусть $\beta$ элемент $S_{1}(\alpha, L)$. Поскольку $\bar{h}(\bar{\beta})=\bar{\beta}+(\bar{\beta}-\bar{\alpha})^{q}$, мы найдем, что $\bar{\beta} \neq \bar{\alpha}$ и $\bar{h}(\beta)=\bar{\alpha}$ тогда и только тогда, когда $(\bar{\beta}-\bar{\alpha})^{q-1}=-1$. Далее рассуждения аналогичны рассуждениям, использованным в случае 1 . Таким образом, в этом случае мы можем сделать такое же заключение об устройстве максимального диска Зигеля, какое мы сделали в случае 1.

Выражаю благодарность научному руководителю профессору А. Хренникову за руководство и консультации, а также благодарю К.-О. Линдала, М. Нильссона, Р. Найквиста из университета Векши за плодотворные обсуждения.

\section{Список литературы}

1. Альбеверио С., Кледен П.К., Хренников А. Ю. Память человека как $p$-адическая динамическая система // ТМФ. 1998. Т. 117. № 3. С. 385-396.

2. Arrowsmith D. K., Vivaldi F. Geometry of $p$-adic sigel discs // Physica D. 1994. V. 71. P. 222-236.

3. Artin E. Algebraic numbers and algebraic functions. N.Y.-L.-Paris: Gordon and Breach Science Publishers, 1967.

4. Benedetto R. Hyperbolic maps in $p$-adic dynamics // Ergodic Theory and Dynamical Systems. 2001. V. 21. P. 1-11.

5. Cassels J. W.S. Local fields. Cambrige: Cambrige Univ. Press, 1986.

6. Khrennikov A. p-adic valued distributions in mathematical physics. Dordrecht: Kluwer Academic Publishers, 1994.

7. Khrennikov A. Non-archimedian analysis: Quantum paradoxes, dynamical systems and biological models. Dordrecht: Kluwer Academic Publishers, 1997.

8. Khrennikov A. Human subconscious as a $p$-adic dynamical system // J. Theor. Biology. 1998. V. 193. P. 179-196.

9. Khrennicov A., Nilson $M$. On the number of cycles of $p$-adic dynamical systems // J. number theory. 2001. V. 90. № 2. P. 255-264.

10. Lang S. Algebraic number theory. 2nd ed. Berlin: Springer-Verlag, 1999.

11. Lidl R., Niderreiter $H$. Introduction to finite fields and their applications. Cambridge: Cambridge Univ. Press, 1994.

12. Lindahl K.-O. On markovian properties of the dynamical systems over $p$-adic numbers // Reports from Växjö University. 1999. V. 8.

13. Nilsson $M$. Cycles of monomial and perturbated monomial $p$-adic dynamical systems // Ann. Math. Blaise Pascal. 2000. V. 7. № 1.

14. Nyqvist R. Dynamical systems in finite field extension of $p$-adic numbers // Reports from Växjö University. 1999. V. 12.

15. Serre J.-P. Local fields. Heidelberg: Springer-Verlag, 1995.

16. Владимиров В.С., Волович И. В., Зеленов Е.И. p-адический анализ и математическая физика. М.: Физматлит, 1994.

17. Volovich I. V. p-adic string // Class. Quant. Grav. 1987. № 4. P. 83-87.

School of Mathematics and System Engineering

Växjö University

Поступило в редакцию

E-mail: Per-Anders.Svensson@msi.vxu.se

17.12 .2003

Перевод с английского Д. В. Прохоренко 